Revue

Revue de l'histoire des religions

de Ihistoire des religions

3 | 2018

Varia

La réforme du calendrier aux conciles de Constance et de Bâle, Corpus édité, traduit et commenté par Olivier de Solan

Paris, CNRS Éditions (Sources d'histoire médiévale publiées par l'Institut de Recherche et d'Histoire des Textes, 42), 2016

Bénédicte Sère

\title{
OpenEdition
}

Journals

Édition électronique

URL : http://journals.openedition.org/rhr/9143

DOI : 10.4000/rhr.9143

ISSN : 2105-2573

Éditeur

Armand Colin

Édition imprimée

Date de publication : 1 septembre 2018

Pagination : 563-566

ISBN : 978-2-200-93188-9

ISSN : 0035-1423

Référence électronique

Bénédicte Sère, "La réforme du calendrier aux conciles de Constance et de Bâle, Corpus édité, traduit et commenté par Olivier de Solan », Revue de l'histoire des religions [En ligne], 3 | 2018, mis en ligne le 01 septembre 2018, consulté le 13 janvier 2021. URL : http://journals.openedition.org/rhr/9143 ; DOI : https://doi.org/10.4000/rhr.9143

Ce document a été généré automatiquement le 13 janvier 2021.

Tous droits réservés 


\section{La réforme du calendrier aux conciles de Constance et de Bâle, Corpus édité, traduit et commenté par Olivier de}

\section{SOLAN}

Paris, CNRS Éditions (Sources d'histoire médiévale publiées par l'Institut de Recherche et d'Histoire des Textes, 42), 2016

\section{Bénédicte Sère}

\section{RÉFÉRENCE}

La réforme du calendrier aux conciles de Constance et de Bâle, Corpus édité, traduit et commenté par Olivier de SolAN, Paris, CNRS Éditions (Sources d'histoire médiévale publiées par l'Institut de Recherche et d'Histoire des Textes, 42), 2016, $25 \mathrm{~cm}, 718$ p., $70 €$, ISBN 978-2-271-09196-3.

1 Le volumineux ouvrage publié par les soins d'olivier de Solan s'inscrit dans le renouveau de l'intérêt des historiens pour le comput, renouveau daté d'une vingtaine d'années, notamment en Allemagne, terre d'élection de cette historiographie. Il se concentre sur la fin du Moyen Âge, le moment où aux conciles de Constance et de Bâle, une réforme du calendrier est sollicitée auprès des pères conciliaires. Assurément, le propos du livre est technique, mêlant le comput médiéval à l'astronomie, calculant les années tropique, sidérale, bissextile, définissant les indictions, les épactes d'une année, les équinoxes et les solstices, les éclipses et les conjonctions, se fondant sur les tables astronomiques et les tables alphonsines, distinguant le mois synodique du mois sidéral, tentant de fixer le terme pascal, les fêtes liturgiques mobiles et les fêtes fixes. En somme, une technicité qu'il fallait historiciser. Ce que ne manque pas de faire l'auteuréditeur du volume, conscient de la difficulté pour un lecteur non-initié. D'où la 
multiplicité des annexes : glossaire, index nominum, index rerum, index fontium, index manuscriptorum, incipitarium.

Le livre s'organise en trois parties qui n'en représentent en réalité que deux : une analyse historique (première et deuxième parties) et des éditions de textes avec leur traduction (troisième partie). Sept textes sont ainsi édités, tous introduits minutieusement avec présentation de leur auteur, datation du texte, précisions contextuelles et description des manuscrits dont les listes se veulent aussi exhaustives que possible. Trois auteurs sont attribués pour cinq textes et deux restent anonymes. Il s'agit, dans l'ordre, des textes suivants: Pierre d'Ailly, Exhortatio super Kalendarii correctione; Anon., Relatio deliberationis prime super materia Kalendarii ; Anon., Avisamentum de correctione Kalendarii ; Hermann Zoest, Tractatus Phase; Hermann Zoest, Phaselexis; Hermann Zoest, Compendium Paschale et Rescriptum ad clerum Coloniensem ; Johannes Keck, Kalendarium. Quant à la célèbre œuvre de Nicolas de Cues, De correctione Kalendarii, bien éditée par ailleurs, elle ne compte pas parmi les œuvres du volume, même si elle est intégrée dans l'analyse historique. Les textes se concentrent donc sur une période allant de 1411 à 1444 , soit la période conciliaire, du concile de Rome (1411-1412) à celui de Bâle (1431-1441-1449), en passant par celui de Constance (1414-1418). L'enjeu porte bien sur la question d'une réforme du calendrier et Olivier de Solan montre dans son analyse historique les cheminements des trois projets présentés $(1435 ; 1436-1437 ; 1437-1440)$, leurs échecs successifs et l'abandon final malgré l'urgence de la réforme. Il examine également les différentes propositions de réforme pour aboutir à la conclusion de la seule viabilité du projet de 1437, lequel n'aboutit qu'un siècle et demi plus tard, sous Grégoire XIII en 1582 pour devenir le calendrier grégorien que nous utilisons actuellement.

3 Reprenons le dossier. Au sens médiéval, le calendrier n'est pas un tableau des jours et des mois de l'année, mais bien un ensemble de règles de calcul du temps : définition du début et de la fin du jour, durée des mois et des années, détermination de certains événements liturgiques ou astronomiques. Calendrier et comput sont équivalents. Le calendrier définit les unités de compte que sont le jour et ses multiples, décrit le cours du soleil et de la lune, et permet le calcul de la date de Pâques, son but principal. Le calendrier dit julien présentait plusieurs défauts qu'il fallait impérativement corriger. Parce qu'il dépendait d'une durée imprécise de l'année solaire et des mois lunaires, l'équinoxe de printemps, normalement fixé au 21 mars, arrivait chaque année de plus en plus tôt, au fil des siècles. De même, les nouvelles lunes, fixées dans le calendrier selon un cycle de 19 ans, se produisaient avec plusieurs jours d'avance. La date de Pâques, cœur et centre de la liturgie chrétienne et de son économie, était fixée en fonction de l'équinoxe de printemps et de la nouvelle lune. Par sa remontée incessante dans le calendrier, elle risquait de ruiner la cohérence liturgique et perdait en signification sotériologique, de sorte que le calendrier en l'état devenait objet de scandale grandissant dans l'Église. Parce qu'il est ecclésiastique - et non astronomique - le calendrier, plus que millénaire, doit être réformé par une institution religieuse. L'occasion des grands conciles $\mathrm{du} \mathrm{Xv}^{\mathrm{e}}$ siècle fut saisie pour inscrire à l'ordre du jour la réforme du calendrier dans le cadre plus général d'une réforme des maux et des abus dans l'Église. L'objectif est de mettre en concordance la date de Pâques avec les phénomènes astronomiques observés. C'est dire que le sujet relève tant des astronomes et computistes que des canonistes. Parmi les propositions de réforme, celle de Pierre d'Ailly nous renseigne sur les modalités de sa production. L'évêque de 
Cambrai, délégué en quelque sorte par une équipe de scientifiques, écrit un traité qu'il fait relire par un astronome de renom, Lubert Hautschild. Puis, il lit publiquement son Exhortatio en mars 1417 en l'église Saint-Paul de Constance. C'est ainsi que, porte-parole de plus savants que lui, il peut introduire le sujet auprès des pères conciliaires. Des échos de ce texte se retrouvent chez Jean Gerson et parmi les contemporains du concile de Bâle. La dimension politique et morale l'emporte sur les considérations techniques. À Bâle, la réforme du calendrier est rattachée à la réforme des mœurs (hoc pertinet ad reformationem morum) ou encore au problème de l'unité des chrétiens, peut-être du fait que la commission de réforme n'était pas formée de spécialistes mais de théologiens et de canonistes influents. Pour autant, la conception technique des projets de réforme devait évidemment revenir à des experts en comput et en astronomie, comme Léonard de Crémone ou d'astronomes extérieurs au concile. Hermann Zoest y a joué un rôle d'expert et de conseiller.

4 Le premier projet de 1435 fut écarté : il proposait un déplacement de trois jours des nombres d'or (cycle lunaire de 19 ans) et une remontée de l'équinoxe de printemps placé le 12 mars. Le second projet, celui de Nicolas de Cues (1436-1437) proposait de supprimer sept jours et d'adopter pour le calendrier lunaire le cycle lunaire des Juifs et des Grecs. C'était un projet canonique plus que scientifique, signe que la question du calendrier était en passe de sortir du cercle des astronomes et des computistes pour devenir l'affaire de l'Église tout entière. Le troisième et dernier projet (1437-1440) conjuguait les deux méthodes de correction proposée - la correction par soustraction et la correction par déplacement - pour aboutir à une méthode mixte : cette technique développée $a u \mathrm{xv}^{\mathrm{e}}$ siècle a permis à la commission du concile de Bâle de produire les deux premiers projets vraiment viables de l'histoire de la réforme du calendrier. Il s'agit donc de supprimer des jours pour corriger le calendrier solaire, et de ne corriger le calendrier lunaire qu'au moyen de déplacements, éventuellement compensatoires, des marques des nouvelles lunes. Au-delà de sa virtuosité compustique, le projet avait l'envergure de se fondre dans le cycle lunaire des Juifs et des Grecs. L'adoption de ce calendrier permet ainsi aux chrétiens de suivre les mêmes années embolismiques que ces derniers. Hermann Zoest insiste sur l'unité retrouvée des trois «langues" (hébraïque, grecque et latine) en ces temps d'efforts pour l'unité dans les années 1440.

5 Si le livre reste, on l'aura compris, d'une lecture ardue pour les non-spécialistes du sujet, l'historien n'en trouvera pas moins matière à réflexion. Par-delà sa dimension technique, la question du calendrier et de sa réforme s'inscrit dans l'horizon plus large d'un enjeu unioniste, d'une part, et canonique, d'autre part. Réajuster la dérive des fêtes mobiles, dont Pâques reste la plus éminente, en tenant compte du comput juif et grec relève d'une décision politique, orientée par l'actualité du temps, celle des négociations avec la délégation grecque venue de Byzance. Elle traduit tout autant la hantise du drame schismatique si présent dans les mémoires. Quant à la dimension canonique de la réforme, elle dit assez le hiatus entre les recherches scientifiques des astronomes et le souci inquiet des canonistes à maintenir la portée symbolique des fêtes liées aux saisons dans leur attachement à la tradition. Ainsi, la genèse de la réforme grégorienne du calendrier, en 1582, révèle par les enthousiasmes qu'elle soulève et les peurs crispées qu'elle suscite, l'état des esprits dans ce $\mathrm{xv}^{\mathrm{e}}$ siècle encore médiéval et déjà frémissant de modernité. 


\section{AUTEURS}

\section{BÉNÉDICTE SÈRE}

Université de Paris Nanterre. 